\title{
Conflicting Processes in the Extinction of Conditioned Taste Aversion: Behavioral and Molecular Aspects of Latency, Apparent Stagnation, and Spontaneous Recovery
}

\author{
Diego E. Berman, Shoshi Hazvi, Jimmy Stehberg, Amir Bahar, and Yadin Dudai ${ }^{1}$ \\ Department of Neurobiology, The Weizmann Institute of Science, Rehovot 76100, Israel
}

\begin{abstract}
The study of experimental extinction and of the spontaneous recovery of the extinguished memory could cast light on neurobiological mechanisms by which internal representations compete to control behavior. In this work, we use a combination of behavioral and molecular methods to dissect subprocesses of experimental extinction of conditioned taste aversion (CTA). Extinction of CTA becomes apparent only 90 min after the extinction trial. This latency is insensitive to muscarinic and $\beta$-adrenergic modulation and to protein synthesis inhibition in the insular cortex (IC). Immediately afterwards, however, the extinguishing trace becomes sensitive to $\beta$-adrenergic blockade and protein synthesis inhibition. The subsequent kinetics and magnitude of extinction depend on whether a spaced or massed extinction protocol is used. A massed protocol is highly effective in the short run, but results in apparent stagnation of extinction in the long-run, which conceals fast spontaneous recovery of the preextinguished trace. This recovery can be truncated by a $\beta$-adrenergic agonist or a cAMP analog in the insular cortex, suggesting that spontaneous overtaking of the behavioral control by the original association is regulated at least in part by $\beta$-adrenergic input, probably operating via the cAMP cascade, long after the offset of the conditioned stimulus. Hence, the performance of the subject in experimental extinction is the sum total of multiple, sometimes conflicting, time-dependent processes.
\end{abstract}

Experimental extinction is the decline in the frequency or intensity of a conditioned response (CR) following the withdrawal of reinforcement (Pavlov 1927). Ample behavioral evidence indicates that experimental extinction does not involve obliteration of the original trace, but rather a relearning process, in which the association of the conditioned stimulus with the absence of the reinforcer comes to control behavior. Multiple types of phenomena support this conclusion: (1) spontaneous recovery: once extinguished, the CR may recover with time without further training (Pavlov 1927); (2) saving (Ebbinghaus 1964): reacquisition of the extinguished behavior requires fewer trials than the original training; (3) reinstatement: exposure to the unconditioned stimulus alone may restore the CR (Rescorla and Heth 1975); and (4) renewal: switching out of the extinction context could cause reemergence of the seemingly extinguished response (Bouton and Swartzentruber 1991). Taken together, these observations indicate that in extinction, the original association is not abolished.

Extinction is one of the most fundamental topics in the experimental psychology of learning and in learning theory

${ }^{1}$ Corresponding author.

E-MAIL yadin.dudai@weizmann.ac.il; FAX 972-8-946-9244.

Article and publication are at http://www.learnmem.org/cgi/doi/ $10.1101 / \mathrm{lm} .53703$. (e.g., Pavlov 1927; Rescorla 1979, 1996; Mackintosh 1983; Bouton 1994). The investigation of the neurobiology of extinction is still in its infancy, yet has intensified in recent years (Christiansen and Schmajuk 1992; Falls et al. 1992; Swank et al. 1996; Wik et al. 1997; LaBar et al. 1998; Lee and Kim 1998; Garcia et al. 1999; Quirk et al. 2000; Weible et al. 2000; Berman and Dudai 2001). Research on extinction is expected to contribute, among others, to our understanding of brain processes and mechanisms by which internal representations compete to control behavior. Furthermore, this research could contribute to the development of drugs capable of specifically modulating the expression of memory items.

Conditioned taste aversion (CTA) is a type of associative conditioning in which the subject learns to associate a taste with delayed malaise (Garcia et al. 1966; Bures et al. 1998). CTA offers advantages for the study of experimental extinction. Learning is fast, and the memory is remarkably robust, yet under the appropriate conditions, CTA extinction could be initiated in a single trial (Berman and Dudai 2001). This facilitates the correlation of cellular and molecular events with the behavioral change, as well as targeted interventions in identified brain areas at discrete phases of the use-dependent behavioral change. The neural substrates of CTA are partially identified (Bures et al. 1998), and some

LEARNING \& MEMORY 10:16-25 @ 2003 by Cold Spring Harbor Laboratory Press ISSN1072-0502/03 \$5.00

$$
\begin{array}{llllllllllllllll} 
& E & A & R & N & I & N & G & \mathbf{Q} & M & E & M & O & R & Y \\
\text { www.learnmem.org } & & &
\end{array}
$$


of the molecular and cellular substrates that subserve learning and consolidation of long-term CTA memory have been identified (e.g., Lamprecht et al. 1997; Rosenblum et al. 1997; Berman et al. 1998, 2000; Gutierrez et al. 1999; Yasoshima et al. 2000). We have recently reported that similarly to consolidation, long-term extinction of CTA requires de novo protein synthesis in the IC, which contains the central taste area, and that the molecular mechanisms of extinction resemble those that subserve the acquisition of extra information about a familiar percept, but differ from those that subserve the acquisition of novel information (Berman and Dudai 2001). In this paper, we set out to identify additional processes and mechanisms that take place in the short- and the long-term phases of CTA extinction, in order to cast additional light on the differences between acquisition of an item in memory and its extinction.

\section{RESULTS}

\section{The Extinction of CTA and the Retention of the Extinguished Trace}

In the absence of extinction training, CTA memory can last for months without significant decay, as can be demon- strated by subjecting different groups of conditioned rats to a single taste-aversion test at various times following training (Fig. 1A). If subjected, however, to an extinction protocol as described under Materials and Methods, CTA memory could be extinguished readily (Fig. 1B). CTA extinction depends on the number of CS-US pairings (Fig. 1B), on the original aversion index (AI) obtained in the given training protocol (Fig. 1B, inset), and on the identity of the taste used as the CS: Conditioned aversion of innately preferred tastes, such as saccharin or polycose, tends to extinguish faster than that of innately aversive ones, such as citric acid or quinine (data not shown).

Once extinction has occurred, it is possible to show that the CTA trace was not eliminated. This we have done by using a saving procedure. Standard CTA training protocols use a strong US, resulting in single-trial acquisition. This makes it impractical to use in saving the same US as in the original training. We have used therefore a protocol that involves a weaker US in retraining. Two groups of rats were first conditioned to avoid saccharin in the usual procedure, using $0.15 \mathrm{M} \mathrm{LiCl}$ i.p. as the US. They were then subjected to an extinction protocol that included three repetitive retrieval tests, each $1 \mathrm{~d}$ apart. On the subsequent $2 \mathrm{~d}$, the rats
A

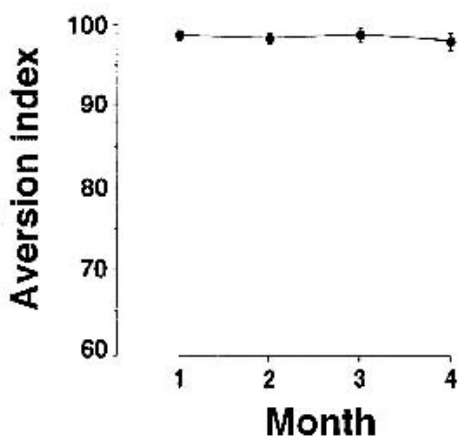

B
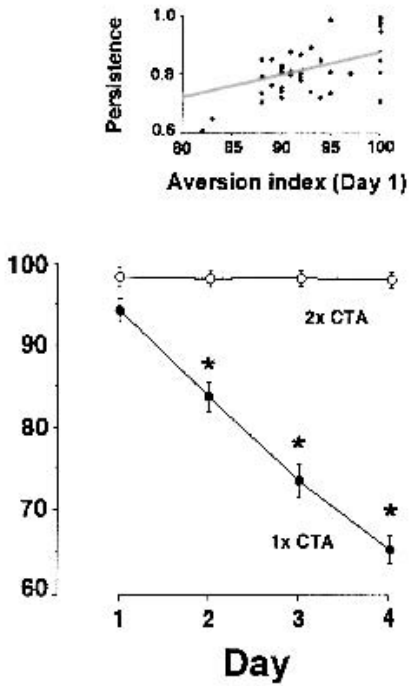

C
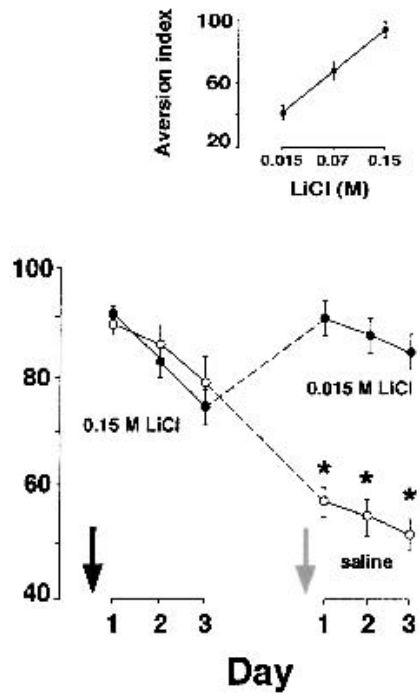

Figure 1 Extinction of CTA as a function of time and US intensity. (A) In the absence of experimental extinction, CTA memory lasts for many months without significant decay. In this panel, each experimental point represents separate experimental groups tested at the indicated time points after CTA training (in this and in the following figures, unless otherwise indicated, $n=8-10$ rats per group). (B) CTA extinction is induced rapidly by providing an opportunity to consume the taste in the absence of the reinforcer (closed circles). However, when the rats were trained once a day for two consecutive days $(2 \times \mathrm{CTA})$, extinction was not detected during the time window covered in this experiment. In this experiment, extinction was measured in the same group of animals. Here and in the figures below, error bars denote SEM, and asterisks indicate significant differences $(p<0.05)$. The significant differences in this figure are between the Al of the control group $(1 \times$ CTA) and the experimental group (2× CTA). (Inset) Persistence of CTA as a function of the initial aversion index. Persistence is depicted as (AI Day 3)/(AI Day 1). In this figure, each data point represents a single animal subjected to a standard CTA training protocol and then tested after $3 \mathrm{~d}$ for 3 consecutive d, once a day. (C) CTA extinction is not unlearning. Rats were conditioned with $0.15 \mathrm{M} \mathrm{LiCl}$ (black arrow), and after $3 \mathrm{~d}$ of extinction, were divided into two groups, one injected i.p. with $0.015 \mathrm{M} \mathrm{LiCl}$ and the other with saline (gray arrow), as described under Results. The group injected i.p. with $0.015 \mathrm{M} \mathrm{LiCl}$, which itself is an ineffective US in naive rats (inset, $\mathrm{Al}<50$, where 50 is the equal preference point), recovered the initial aversion (closed circles), whereas the group injected with saline showed further extinction (open circles).

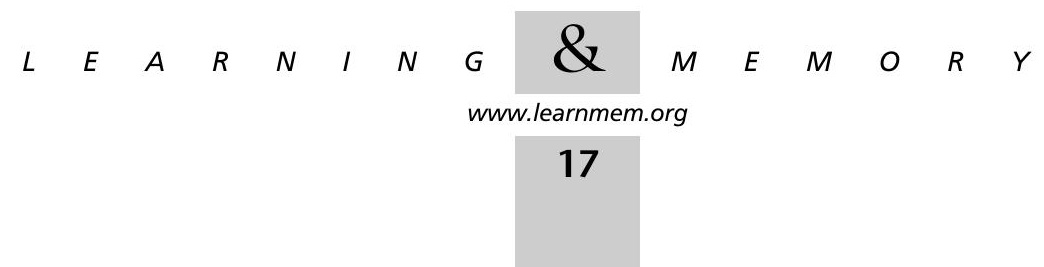


received water only. On the day afterwards, they were reexposed to saccharin. Then, $40 \mathrm{~min}$ later, one group was injected i.p. with a dose of $\mathrm{LiCl}$ that is ineffective in inducing CTA in naive rats (Fig. 1C, inset), whereas the other group was injected i.p. with saline as control. Whereas the control group continued to extinguish the CTA to saccharin, the weak-US rats regained the original aversion score, as if they had never been subjected to the extinction procedure (Fig. 1C). This saving of the original aversion by the weak US was not the result of a generalized sensitization to the US. This was determined by training a different group first on saccharin, using the routine strong-US procedure, followed by training with another taste, polycose, using the weak US. This weak-US training did not lead to CTA to polycose (data not shown). Hence, the finding that the original CTA reappeared by retraining with a weak US indicates that the original associative trace was never abolished.

\section{The Short and the Long of CTA Extinction}

Extinction in CTA is commonly determined by subjecting the conditioned subjects to the unreinforced CS $24 \mathrm{~h}$ apart (e.g., Brooks et al. 1999; Berman and Dudai 2001). We wished, however, to determine the processes and mechanisms of shorter phases of extinction. We have therefore tested extinction at $\geq 30 \mathrm{~min}$ after retrieval (Fig. 2 ; the first retrieval test of CTA, in which the CS is presented for the first time after training in the absence of reinforcer, is hence $t=0$ for extinction). To our surprise, we found that extinction develops as a step function: the extinguished performance was undetected for the first $90 \mathrm{~min}$ after retrieval. The basic effect was replicated with other tastes, polycose and glycine (data not shown).

What could be the reason for this short-term latency? The first possibility that comes to mind is that the rat's brain waits for some time, throughout which food poisoning might develop, before deciding whether this food is safe or not. This possibility should, indeed, be considered as an explanation for the lack of immediate extinction performance of CTA. But there is also at least one other possibility, that the mere experience of retrieval of an aversive stimulus is itself aversive and could affect subsequent behavior. To test this hypothesis, we have subjected three groups of rats (Groups I, II, III in Fig. 3A) to CTA on Taste 1 (saccharin). We then conditioned one of the groups (Group I) to avoid another Taste 2 (polycose), which is perceived by the rat as different from Taste 1 (the fact that the rats perceive polycose as different from saccharin is evident from the weak-US saving experiment described above; similar conclusions concerning the discriminability of the tastes were obtained from latent inhibition experiments by A. Bahar and Y. Dudai, unpubl.). Subsequently, we continued the extinction trials on Taste 1 in all three groups. The rats in Group I, which were trained on CTA to Taste 1 , then on CTA to Taste 2 , and then trained to extin-

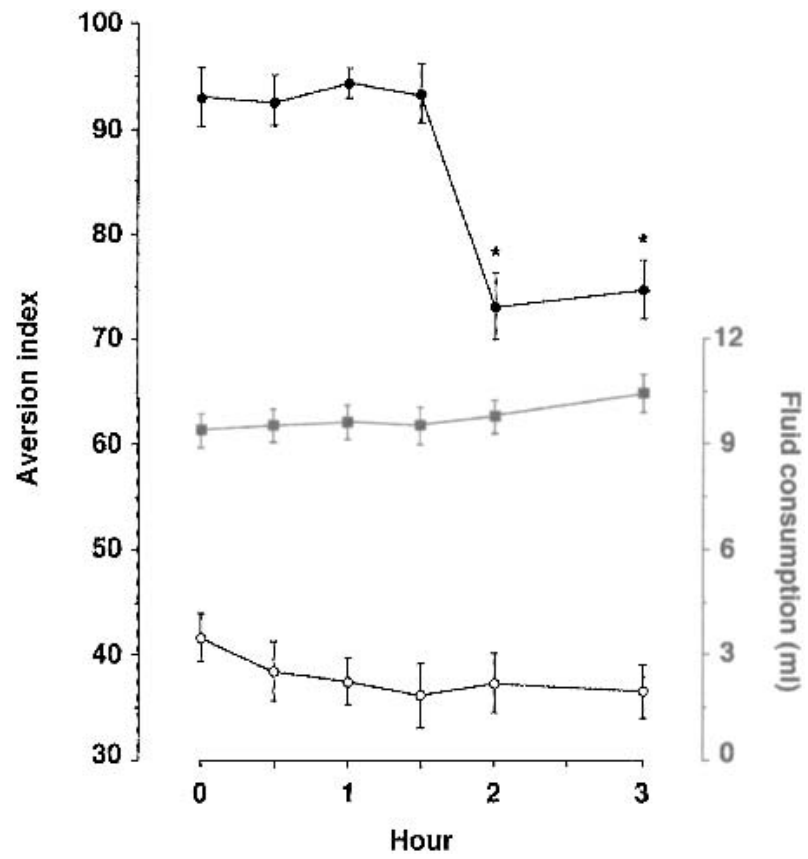

Figure 2 Kinetics of CTA extinction immediately after retrieval. Groups were trained as described under Materials and Methods. Then, $3 \mathrm{~d}$ later, they were subjected to a retrieval trial in the absence of reinforcer $(t=0)$. This was followed by a second retrieval trial at the indicated times. Each group of rats was tested on a single time point only in addition to $t=0$, that is, each time point in the graph represents a separate group. Extinction becomes apparent only at $2 \mathrm{~h}$ after time zero (closed circles). Control groups that were injected i.p. with saline instead of $\mathrm{LiCl}$ during CTA training did not show aversion (open circles). The amount of liquid consumed did not vary significantly between the groups and the different time points (closed gray squares, data of the experimental groups).

guish on Taste 1 , regained their avoidance to Taste 1 immediately after retrieval of CTA to Taste 2 (Fig. 3B). In contrast, the rats that were conditioned only on Taste 1 , then subjected to extinction trials on the same taste, while being exposed in between to Taste 2 (Group II), or the rats that were conditioned only on Taste 1 , then subjected to extinction trials on the same taste, and not exposed to Taste 2 at all (Group III), did not show a similar rebound of avoidance (Fig. 3B). The rebound of the extinguished memory of CTA of Taste 1 in Group I was short-lived, and resembled in its kinetics the time course of the delayed extinction shown in Fig. 2. It could be suggested, therefore, that the mere experience of retrieval of the memory of aversion generates a short-term aversive trace, which could block or mask the immediate development of extinction.

We then proceeded to find out whether we can identify molecular correlates of the extinction latency. Toward this end, we have tested several ligands that were previously shown by us to inhibit either acquisition or long-term extinction of CTA (Berman et al. 2000; Berman and Dudai 2001), for their effect on the short-term extinction. When microinfused into the IC immediately after the first retrieval

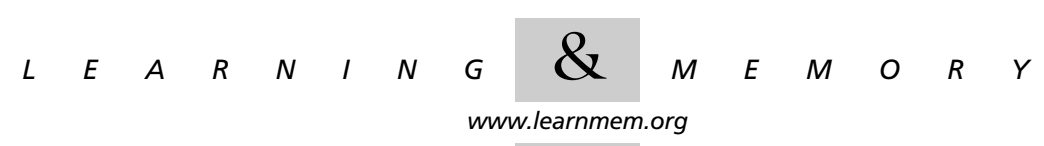


A

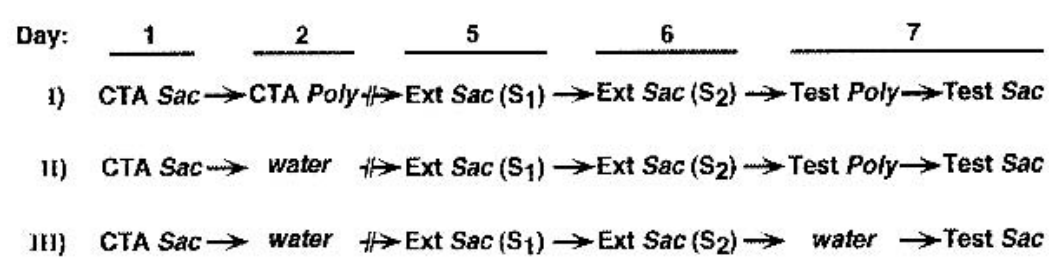

B

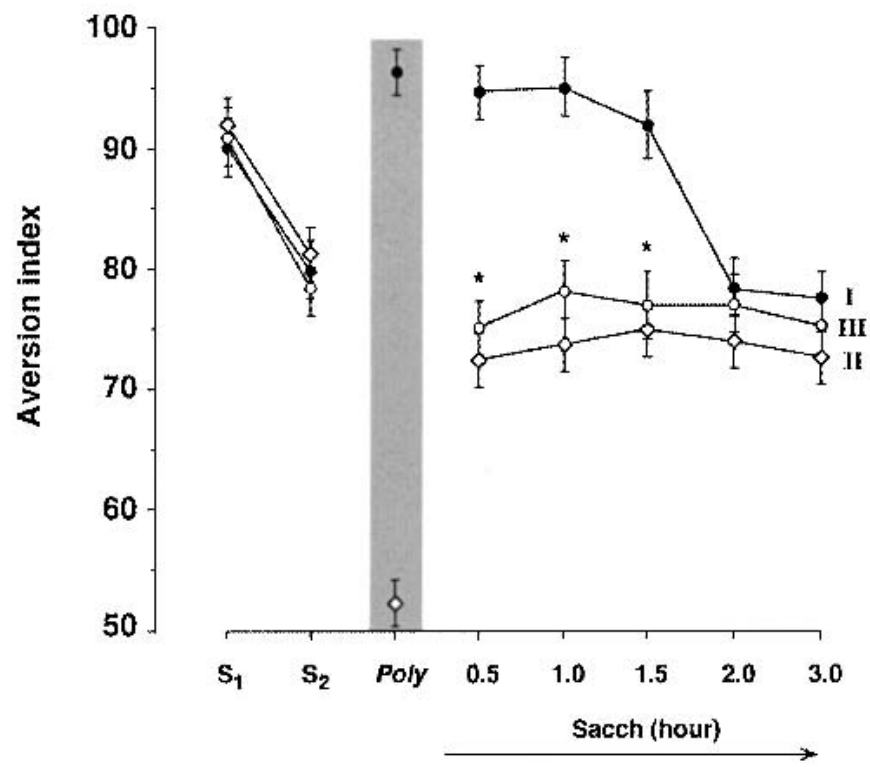

Figure 3 The effect of retrieval of aversion to a taste on the extinction of aversion to another taste. $(A)$ Experimental design. Three groups of rats were trained on CTA to saccharin (Sac). On Day 2, Group I was also trained on CTA to polycose (Poly), whereas Groups II and III received water only. All the groups received water for the subsequent $2 \mathrm{~d}$. On Days 5 and 6, all the groups were subjected to retrieval tests of the saccharin aversion, which partially extinguished $\left(\mathrm{S}_{1}, \mathrm{~S}_{2}\right)$. On Day 7 , Groups I and II were tested for polycose aversion (Poly, shaded area), whereas Group III received water. The groups were then divided each into subgroups, and each of the subgroups ( $n=8-10$ each) was tested for the aversion to saccharin at the times indicated in $B$; hence the data for each time point on the axis labeled Sacch (Saccharin) in $B(0.5-3 \mathrm{~h})$ represent rats tested only once in this stage of the experiment. $(B)$ The latency of extinction (see Fig. 2 and text) was observed in Group I only (closed circles). Group II (open diamonds); Group III (open circles). As in the data presented in Figure 2, total liquid consumption did not vary significantly among the groups (data not shown). Asterisks denote significant differences $(p<0.05)$ in Groups II and III versus Group I.

trial (i.e., $t=0$ for extinction), none of these drugs affected the extinction latency (Fig. 4). Interestingly, whereas the protein synthesis inhibitor anisomycin started to block extinction of CTA already at $2 \mathrm{~h}$ after retrieval, it had no effect on CTA memory when tested at $2 \mathrm{~h}$ (Fig. 4, inset). This indicates that the involvement of protein synthesis in acquisition and extinction is not identical. We have previously reported (Berman et al. 2000; Berman and Dudai 2001) that neither anisomycin, nor the $\beta$-adrenergic antagonist propranolol or the muscarinic antagonist scopol- amine, when microinfused into the IC, serve as a reinforcer in CTA. Furthermore, none of these drugs significantly affected the total fluid consumption in the shortterm extinction experiments (data not shown). We conclude, therefore, that the early postretrieval phase, in which no extinction performance is apparent, is independent of protein synthesis and $\beta$-adrenergic and cholinergic modulation. Once the latency stage is over, however, extinction at $>2 \mathrm{~h}$ is blocked by inhibition of protein synthesis and $\beta$-adrenergic transmission (Fig. 4).

\section{The Effect of Massed Versus Spaced Protocols of Extinction}

We found that the kinetics of extinction are dependent on whether a massed- or spaced-extinction protocol is used. Rats subjected to a single retrieval session in the absence of the reinforcer and then tested for CTA $24 \mathrm{~h}$ later showed evidence for the progression of extinction (Figs. 1B,C and 5A). If, however, instead of a 24-h spaced extinction protocol, a massed-extinction protocol was used, in which the rats were subjected to the unreinforced retrieval every $2 \mathrm{~h}$, the pattern of kinetics of extinction differed: Extinction proceeded in between trials, but no further extinction was evident $>1 \mathrm{~d}$ after the last extinction trial (Fig. 5). Microinfusion of anisomycin immediately after a retrieval trial truncated the progression of extinction in the massed protocol (Fig. 5A), and had no effect on the prolonged apparent stagnation phenomena (Fig. 5B).

\section{Spontaneous Recovery of CTA Extinction}

What underlies the long-term apparent stagnation in extinction following the last trial in the massed-extinction protocol? To gain insight into the underlying process(es), the progression of extinction was assayed using higher temporal resolution. This analysis unveiled in the massed-extinction protocol immediate progression of extinction for $\sim 2 \mathrm{~h}$, followed by spontaneous recovery, yielding the net long-term apparent stagnation observed afterwards (Fig. 6A). No spontaneous recovery was detected after a single extinction trial in the spaced-extinction protocol (Fig. 6A). The spontaneous recovery seen after massed extinction could be prevented by microinfusion into the IC, immediately after the

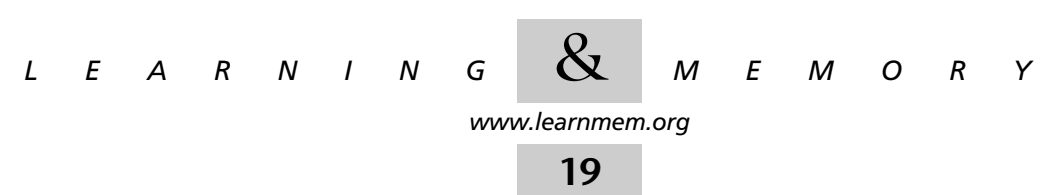




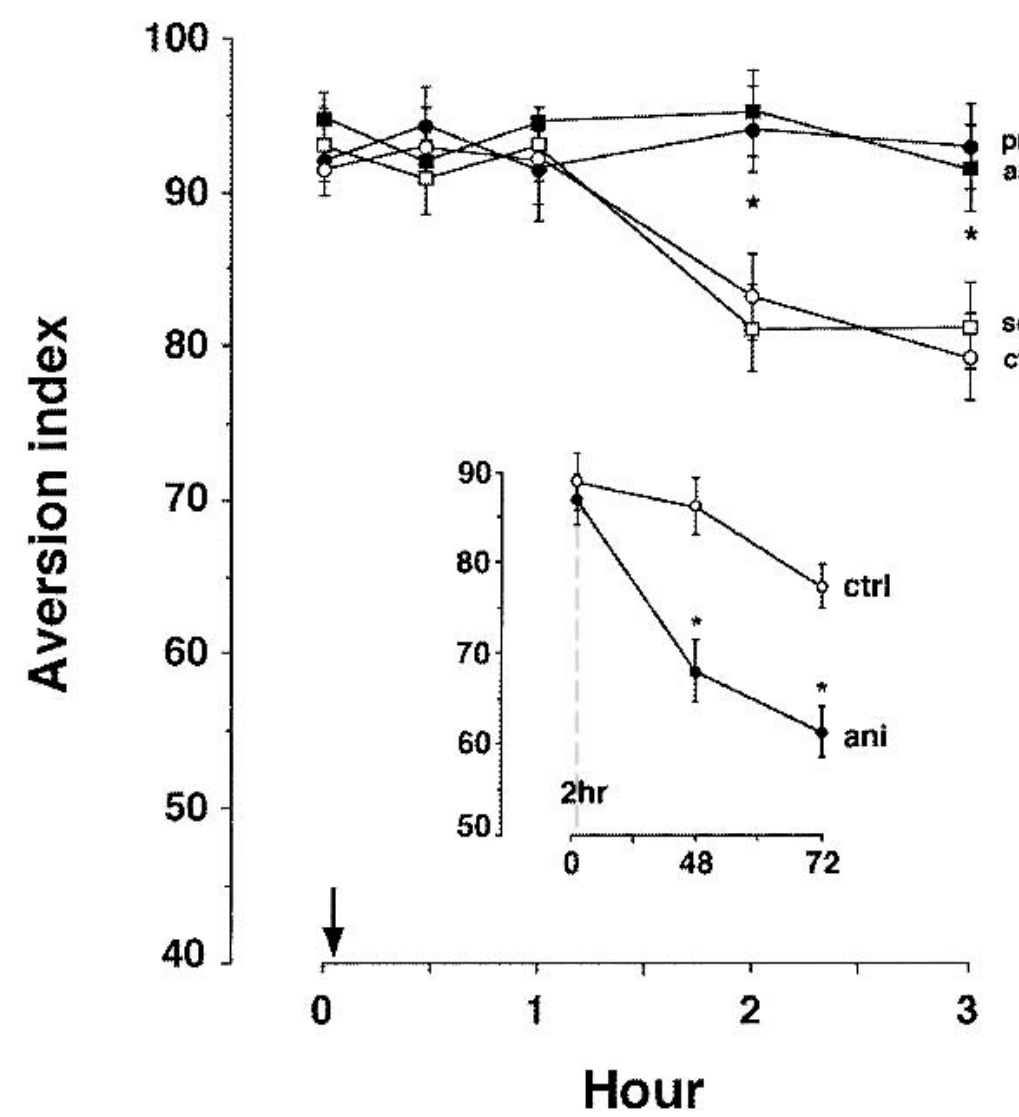

Figure 4 The effect of specific inhibitors on short-term extinction of CTA memory. Drugs were microinfused into the IC bilaterally as described in Materials and Methods (i.e., immediately after the first retrieval trial, arrow). Each experimental point represents a separate group of animals. (prop) Propranolol ( $20 \mu \mathrm{g}$, closed circles); (ani) anisomycin $(100 \mu \mathrm{g}$, closed squares); (scop) scopolamine ( $50 \mu \mathrm{g}$, open squares); (ctrl) control (vehicle only, open circles). Asterisks denote significant differences $(p<0.05)$ between the $\mathrm{Al}$ of the propranolol and anisomycin groups versus the control group. (Inset) Anisomycin (ani, closed circles) microinfused into the IC 20 min before CTA training does not block short-term CTA memory tested at $2 \mathrm{~h}$ after conditioning. In this experiment, the same groups of animals were tested over time.

last extinction trial, of a $\beta$-adrenergic agonist, clenbuterol, or of the cAMP stable analog 8-Br-cAMP (Fig. 6B). In contrast, the $\beta$-adrenergic antagonist, propranolol, had no effect either on the kinetics or on the level of extinction (Fig. 6B).

\section{DISCUSSION}

The study of experimental extinction is potentially capable of opening a window into the dynamics of internal representations of an item and its associations, and into the control of behavior by these internal representations. Although extinction results in diminution of a conditioned response, it is not a consequence of the unlearning of the conditioned association; multiple phenomena, including spontaneous recovery, saving, renewal, and reinstatement, indicate that the original association is not obliterated. Rather, extinction results in the formation of a new internal representation that takes control over behavior. Given the appropriate conditions, the original, apparently extinguished conditioned association can regain behavioral control, while the learned extinguished behavior is "forgotten" (Bouton 1994). CTA offers significant advantages for the investigation of the neurobiological bases of this and other manifestations of extinction, because it involves fast acquisition, and robust yet extinguishable memory, in partially identified neural circuits.

The ability to extinguish CTA is a function of multiple parameters, including the innate palatability of the taste, and the initial aversion index after training. Once extinction has taken place, three phenomena merit special consideration: (1) that the initial CTA associative trace is not erased; (2) that extinction is not instantaneous; and (3) that the kinetics of extinction are markedly affected by whether the extinction protocol involves spaced or massed extinction.

That the associative trace is not unlearned is congruent with the prevailing views of extinction (e.g., Rescorla 1996). In the present study it is evident from the observation that whereas a low concentration of the US, LiCl i.p., did not induce aversion in naive rats, it did so in rats that underwent experimental extinction. Hence, after extinction, the rats still retained at least some representation of the original aversive association, and the retraining with the weak US, which had no effect on naive rats, led to the quick reappearance of this association.

That extinction is not instantaneous, but rather becomes apparent only after a postretrieval latency of many minutes, could be explained by single- or multiple-trace types of explanation. A single-trace, parsimonious explanation, which is inferred from the properties of CTA but for which we have no direct experimental evidence, is that the rat's brain is programmed to wait before a decision is taken concerning the safety of a taste, because in nature it takes time for food poisoning to develop. Another interesting possibility, which was not explored in this study, is that the aforementioned latency results from the rats remembering not only the CS-US association itself, but also that the interstimulus interval (ISI) was long; however, it is noteworthy that the ISI used in our CTA training protocol was $40 \mathrm{~min}$, whereas the latency was at least twice as long. Furthermore, postexperience latency in conditioned performance is not necessarily related to the distinc-

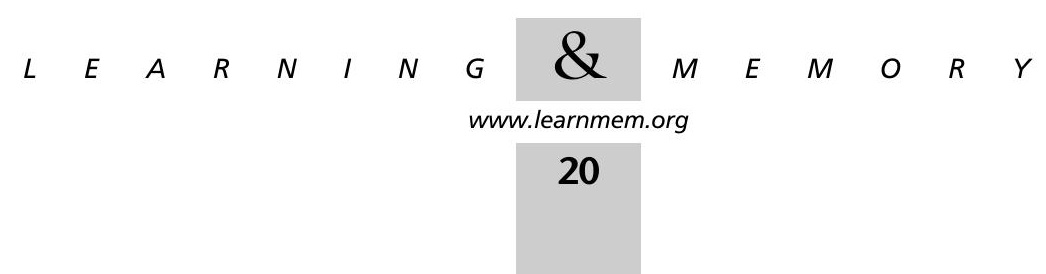


A

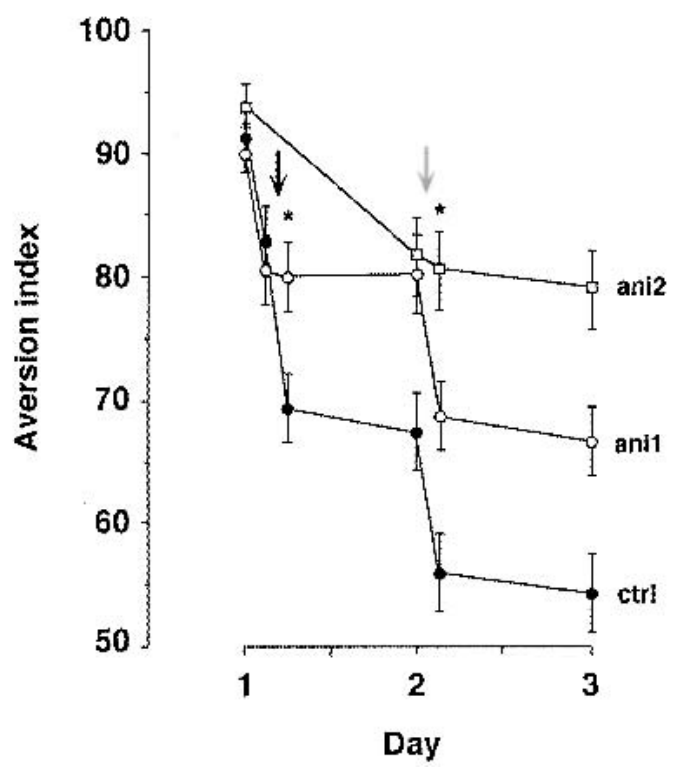

B

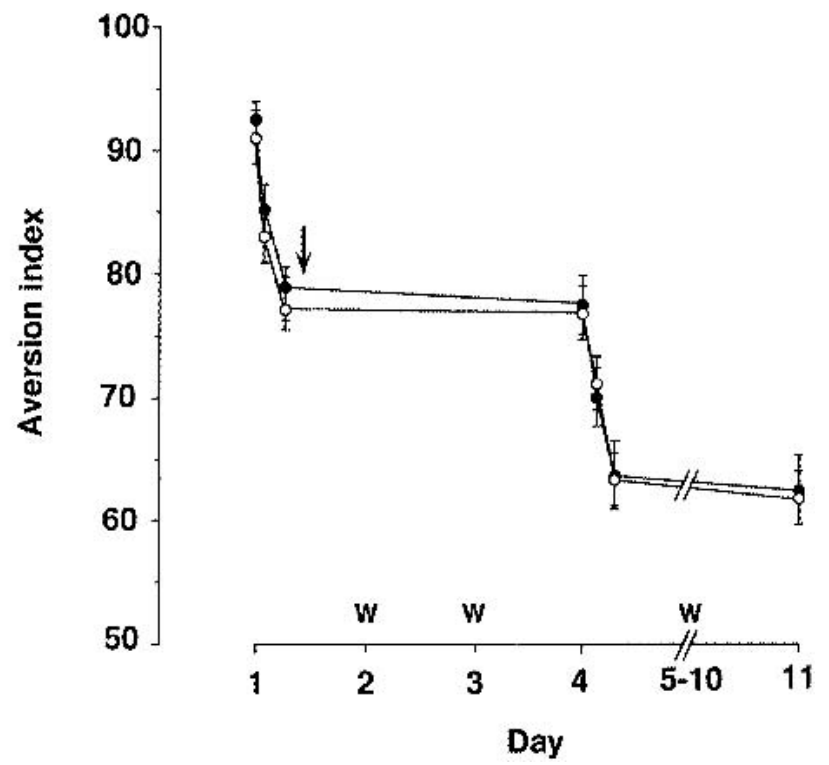

Figure 5 Kinetics of massed and spaced extinction. (A) Marked extinction develops rapidly in animals subjected to a massed-extinction protocol, that is, multiple-extinction trials $2 \mathrm{~h}$ apart (closed circles, ctrl). Anisomycin microinfused into the IC immediately after the second extinction trial, regardless of whether this second extinction trial is on the same day (black arrow, ani1) or a day later (gray arrow, ani2), blocks further extinction. Note that when animals are exposed to massed extinction (ctrl and ani1), no further extinction is detected $24 \mathrm{~h}$ later. Asterisks in this figure denote significant difference $(p<0.05)$ between the ani1 and the ctrl group on Day 1 , and between ani1 and ani2 on Day 2. (B) Extinction does not proceed further when tested $>1$ day after a massed-extinction protocol on Day 1 . Anisomycin (open circles) had no effect on this apparent stagnation of extinction when microinfused into the IC immediately after the last extinction trial in the massed-extinction protocol. (Closed circles) Control animals; (W) water. No statistical significant difference was observed between the control and the anisomycin groups.

tive attributes of CTA such as prolonged ISI; a learning process with an incubation period of $\sim 1 \mathrm{~h}$ has been proposed in relearning of an incompletely acquired avoidance response, in which the ISI was a few seconds only (Kamin 1957). A candidate multiple-trace explanation for our findings is that the mere experience of retrieval of an aversive stimulus, even in the absence of the reinforcer, is itself reinforcing, and that it summates (e.g., Aydin and Pearce 1997) with the extinguishing aversion of the conditioned stimulus, or temporarily reinstates it. Regardless of whether the extinction latency in our system is due to single- or multiple-trace processes, it is short-lived only, and is not subserved by $\beta$-adrenergic receptors, muscarinic cholinergic receptors, or protein synthesis. The extinction observed after this latency is, however, protein-synthesis-dependent. This finding is congruent with the view that extinction involves the consolidation of a new trace (Berman and Dudai 2001), but that the time course of this consolidation is different from that of the consolidation of a newly acquired trace, as the latter becomes dependent on protein synthesis only at a later stage (cf. Fig. 4 with Berman and Dudai 2001, Fig. 2A).

Our data indicate that the apparent stagnation of extinction following the massed-extinction protocol masks partial spontaneous recovery (Fig. 6A). Spontaneous recovery, that is, the recovery of the conditioned response after extinction in the absence of further training (Pavlov 1927), has been demonstrated over the years in many types of Pavlovian and instrumental conditioning, and more recently also in CTA (Rosas and Bouton 1996; Brooks et al. 1999). Spontaneous recovery is of great interest to learning theory because it might cast light on brain processes and mechanisms by which one internal representation (the CS-no reinforcer association) gives way with time to the control of behavior by another representation (the original CS-reinforcer association). In the case of CTA, it could hence unveil how the rat makes up its mind on whether to practically abandon the dislike of a taste or retain it. Spontaneous recovery is also of marked practical interest, because it might model relapse after therapeutic extinction of behavioral disorders (Bouton and Swartzentruber 1991). Multiple explanations have been proposed for spontaneous recovery; an influential one is that spontaneous recovery represents retrieval failure, resulting from the change in extinction context (Bouton and Swartzentruber 1991; Kraemer and Spear 1992), and that this context includes time itself (Bouton 1993).

Neurobiological explanations of the mechanisms of spontaneous recovery of CTA should accommodate four observations (Fig. 6): (1) Spontaneous recovery is dependent on the extinction protocol. In our study it was observed after massed-extinction trials, but not after a single

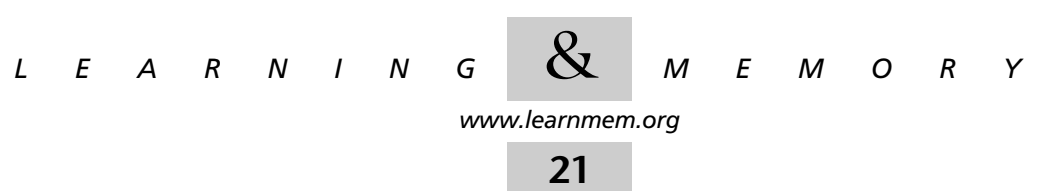


A

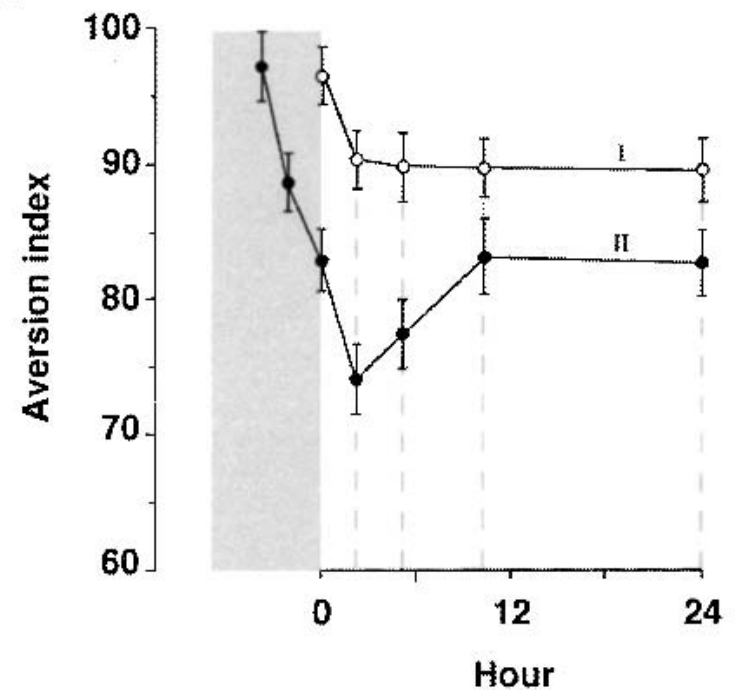

B

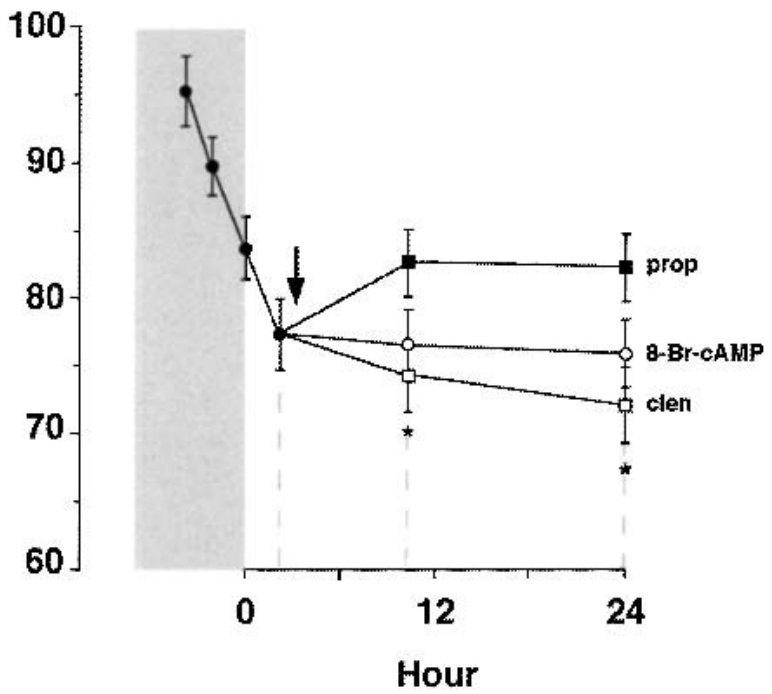

Figure 6 Spontaneous recovery of CTA. (A) Two groups of rats were subjected to CTA training on saccharin. Group I (open circles) was tested once $3 \mathrm{~d}$ after training. Group II (closed circles) was also tested $3 \mathrm{~d}$ after training, but on three successive trials, $2 \mathrm{~h}$ apart (i.e., massed training). The groups were then divided into subgroups, and each of the subgroups was subjected to a single extinction test at the indicated time points; that is, each of the data points at $t>0$ represents a separate group. Only Group II, which was subjected to a massed-extinction protocol, displayed spontaneous recovery (closed circles, $>2$ h). (B) Spontaneous recovery of CTA can be blocked by the microinfusion (black arrow) into the IC of (clen) clenbuterol ( $1 \mathrm{ng}$, open squares), and 8-Br-cAMP ( $86 \mathrm{ng}$, open circles), but not by (prop) propranolol (20 $\mu \mathrm{g}$, closed squares). Asterisks here denote significant differences $(p<0.05)$ in the $\mathrm{Al}$ of the clenbuterol and the 8 -Br-cAMP groups versus the propranolol group.

extinction trial in the spaced-extinction protocol. One could argue that the lack of spontaneous recovery after a single extinction trial is due to the fact that extinction has not proceeded far enough to allow spontaneous recovery to take place. Although this might be possible, it is noteworthy that we found no evidence for immediate recovery of the original conditioned association of CTA in a spaced-extinction protocol even when extinction has proceeded to much lower values of aversion than those achieved in the present massed-extinction protocol (cf. Fig. 6A to Berman and Dudai 2001, Fig. 2). Spontaneous recovery, however, was observed $18 \mathrm{~d}$ after 3 consecutive $\mathrm{d}$ of single-trial extinction training (Brooks et al. 1999). It is hence possible that the difference in the outcome of massed versus spaced protocols is in the kinetics of spontaneous recovery, which is faster after massed extinction, rather than in the mere ability of the extinction protocol to invoke spontaneous recovery. (2) Spontaneous recovery is fast and reaches a plateau within a few hours. (3) Spontaneous recovery is incomplete (see also Rosas and Bouton 1996). (4) Immediately after an extinction trial in the massed-extinction protocol, $\beta$-adrenergic stimulation in the IC, probably acting via the cAMP cascade, blocks spontaneous recovery.

The difference in the effect of massed versus spaced extinction brings to mind the difference between massed and spaced training in general (Green 1989; Pereyra et al. 2000). On the mechanistic level, it has been proposed that in the acquisition of memory, the intensity of the teaching signal(s), and the particular kinetics and interaction of the specific components of the intracellular signal transduction cascades that are set in motion by these signals, determine whether consolidation of long-term memory will be accomplished or not. Particularly, the kinetics of activation and persistence of members of the CREB family of transcription factors have been proposed to explain why massed training is inferior to spaced training, why under certain conditions instant consolidation occurs (e.g., flashbulb memory), and why certain molecular interventions render massed training as strong as spaced training (Yin et al. 1995). Along these lines, one might consider the possibility that augmenting the $\beta$-adrenergic input in cortex after retrieval in the extinction training tilts the balance of signal transduction activation, via the cAMP cascade, so that the new representation remains more stable. This results in truncation of spontaneous recovery at the behavioral level. Because the $\beta$-adrenergic antagonist did not affect spontaneous recovery, we may assume that in situ, the postulated $\beta$-adrenergic input in cortex in the CTA extinction trial or immediately afterwards is short-lived. Further analysis is required, however, to determine how this $\beta$-adrenergic-dependent process is related to the protein-synthesis-dependent process(es) that are required to establish long-term extinction (Berman and Dudai 2001), and which additional molecular mechanisms are involved in regulating the fate of the extinguishing trace

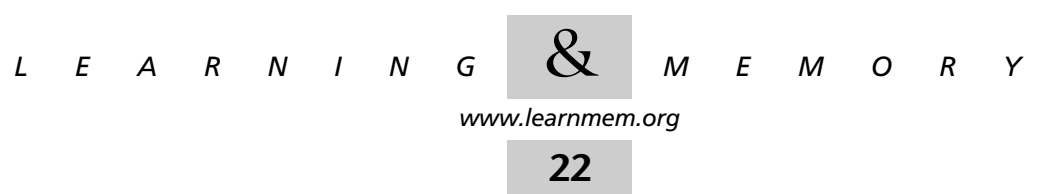


after spaced or massed extinction. Such analysis should take into account the observation that anisomycin after massed extinction does not alter the apparent long-term stagnation (Fig. 5B), and hence the postulated net spontaneous recovery of the extinguished CTA memory. Data from previous analysis of the role of protein synthesis in long-term CTA extinction cannot be adapted as is into situations that involve massed extinction, because these data refer to the effect of protein synthesis blockade in the IC after a single extinction trial (Berman and Dudai 2001), whereas massed extinction, as noted above, is likely to modify the kinetics of the protein-synthesis-dependent processes.

Still another possibility, in line with the aforementioned retrieval theory of spontaneous recovery, is that the drug-induced boost of $\beta$-adrenergic stimulation immediately after retrieval reinstates a context similar to that of the extinction experience. Interestingly, one class of attempts to explain the advantage of spaced versus massed training is "contextual variability theories," which propose that spacing allows richer variability and exploitation of the context, which increases the number of retrieval cues and therefore facilitates retrieval (Green 1989); CTA acquisition and extinction are strongly context-dependent (e.g., Bonardi et al. 1990; A. Desmedt and Y. Dudai, unpubl.), and the $\beta$-adrenergic stimulation could be one of the internal contextual cues. The fact that the $\beta$-adrenergic stimulus is administered after and not before retrieval should not pose a major problem: The association of taste with internal states tolerates long interstimulus intervals. It is also noteworthy that posttraining $\beta$-adrenergic modulation was recently found to affect consolidation of memory in other systems (e.g., McGaugh 1966; Przybyslawski et al. 1999).

The aforementioned possibilities are not mutually exclusive. Whatever the explanation is, the ability to identify molecular fingerprints of spontaneous recovery, similar to the distinct molecular fingerprints of acquisition and extinction of CTA (Berman and Dudai 2001), could contribute to our understanding of the molecular and cellular processes that subserve switches in the control of behavior by internal representations of multiple, sometimes competing, associations of an item.

\section{MATERIALS AND METHODS}

\section{Behavioral Procedures}

Male Wistar rats ( $\sim 60 \mathrm{~d}$ old, $\sim 250 \mathrm{~g})$ were caged individually at $22^{\circ} \pm 2^{\circ} \mathrm{C}, 12 / 12 \mathrm{~h}$ light/dark cycle, in opaque plastic boxes $(30 \times 35 \times 17 \mathrm{~cm})$, with a metal cover fitted with openings for delivering food and liquid. The rats remained in their home box throughout the study, and were removed only briefly for weighing and drug microinfusions, as detailed below. The behavioral protocol of CTA was as detailed in Berman et al. (2000), with minor modifications. Unless otherwise indicated, sodium saccharin $(0.1 \%$ [w/v]) was the conditioned stimulus (CS), and $\mathrm{LiCl}$ i.p. (0.15 M, $2 \%$ body weight) the toxicosis-inducing agent. Rats were first sub- jected to $24 \mathrm{~h}$ of water deprivation and then pretrained to get their water ration once a day (during 3-4 consecutive d) for $10 \mathrm{~min}$, from a pipette containing $10 \mathrm{~mL}$ of tap water. All the drinking and testing procedures were done in the aforementioned home box. On the conditioning day, rats were allowed to drink the saccharin solution $(10 \mathrm{~mL})$ instead of water for $10 \mathrm{~min}$, and $40 \mathrm{~min}$ after the offset of the drinking period were injected with $\mathrm{LiCl}$ i.p. Then, $3 \mathrm{~d}$ after training, rats were tested for $10 \mathrm{~min}$ by presenting them with six pipettes, each of which containing $5 \mathrm{~mL}$ of liquid (single test per day) or $3 \mathrm{~mL}$ of liquid (multiple tests per day). Three of the pipettes contained water and the other three saccharin. In this test situation, the rats explore, sample, and subsequently consume or avoid the contents of several pipettes, none of which contains by itself sufficient liquid to satiate their thirst. This test method diminishes potential confounding effects of intensive motivation to drink, that could exist in single-bottle tests, by permitting the rat to make a preference choice and still satisfy its thirst. Conditioned rats preferred water to saccharin at a ratio of 9:1 on their first postconditioning test in this multiple-choice situation, whereas unconditioned rats preferred saccharin to water. The results were quantified by an aversion index ( $\mathrm{AI})$, defined as $\mathrm{AI}=[$ (milliliters of water)/(milliliters of water + milliliters of saccharin) $] \times 100$ consumed in the test. During extinction, animals were presented with the same multiple-choice situation as above at the times indicated under Results. In the experiments depicted in Figures 1A, 2-4, each group of animals was tested for extinction only once, at the indicated time point after the first retrieval session. Each AI measurement in these experiments hence represents different experimental groups. In the "massed-extinction" protocols (Figs. 5 and 6), animals were presented with the extinction trials on the same day, $2 \mathrm{~h}$ apart, and sometimes later on consecutive or spaced days as indicated under Results. In a preliminary experiment, we tested the effect of eight repetitive extinction tests, $2 \mathrm{~h}$ apart, on the amount of liquid consumed on each test by rats subjected to the aforementioned pretraining and CTA training protocols. The amount of liquid consumed did not vary significantly between the first $(9.4 \pm 0.9$ $\mathrm{mL})$ and the fourth trial $(9.6 \pm 0.6 \mathrm{~mL})$, then started to decline, reaching $1.4 \pm 0.2 \mathrm{~mL}$ on the eighth test ( $n=5$ animals each). We have therefore used a maximum of four trials in those experiments in which extinction was tested by subjecting the same group of rats to repetitive retrievals $2 \mathrm{~h}$ apart (Figs. 5 and 6).

\section{Surgery and Local Microinfusions}

Rats were anesthetized with $4.8 \mathrm{~mL} / \mathrm{kg}$ Equithesin $(2.12 \%[\mathrm{w} / \mathrm{v}]$ $\mathrm{MgSO}_{4}, 10 \%[\mathrm{v} / \mathrm{v}]$ ethanol, $39.1 \%[\mathrm{v} / \mathrm{v}]$ propylene glycol, $0.98 \%$ [w/v] sodium pentobarbitone, $4.2 \%[\mathrm{w} / \mathrm{v}]$ chloral hydrate), restrained in a stereotaxic apparatus (Kopf), and implanted bilaterally with a stainless steel guide cannula ( 23 gauge) aimed $1.0 \mathrm{~mm}$ above the gustatory neocortex (anteroposterior, $+1.2 \mathrm{~mm}$ relative to bregma; lateral, $\pm 5.5 \mathrm{~mm}$; ventral, $5.5 \mathrm{~mm}$; Paxinos and Watson 1998). The cannulae were positioned in place with acrylic dental cement and secured by two skull screws. A stylus was placed in the guide cannula to prevent clogging. Animals were allowed 1 wk to recuperate before being subjected to experimental manipulations. The stylus was removed from the guide cannula, and a 28-gauge injection cannula, extending $1.0 \mathrm{~mm}$ from the tip of the guide cannula, was inserted. The injection cannula was connected via PE20 tubing to a Hamilton microsyringe driven by a microinfusion pump (CMA/100; Carnegie Medicin). Cannula placement was verified by infusing $1 \mu \mathrm{L}$ of India ink in a random sample of 20 rats after the completion of the behavioral experiments; the cannula place-

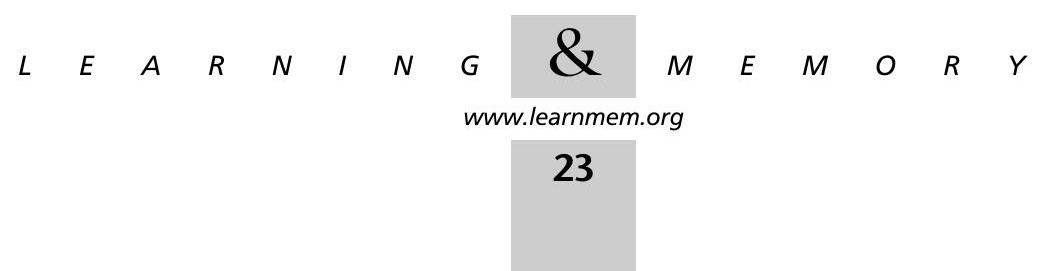


ment and the diffusion sphere of the microinfused solution were as reported in Figure 1A in Berman and Dudai (2001). Microinfusion of anisomycin $(100 \mu \mathrm{g})$, scopolamine $(50 \mu \mathrm{g})$, propranolol $(20 \mu \mathrm{g})$, clenbuterol ( $1 \mathrm{ng}$ ), and 8-Br-cAMP ( $86 \mathrm{ng}$; Sigma) was performed bilaterally in a $1-\mu \mathrm{L}$ volume per hemisphere delivered over $1 \mathrm{~min}$. The injection cannula was left in position before withdrawal for an additional $1 \mathrm{~min}$ to minimize dragging of the injected liquid along the injection tract. The doses of anisomycin, scopolamine, and propranolol were according to Rosenblum et al. 1993; Naor and Dudai 1996; and Berman et al. (2000); and those of clenbuterol and 8-Br-cAMP according to Ferry et al. (1999).

All the drugs used in this study were tested in previous studies, or in preliminary experiments for this study, for their potential ability to serve as a negative or positive reinforcer in CTA training, or their potential effect on the sensorimotor capabilities required to perform the CTA response normally. This was done by substituting $\mathrm{LiCl}$ in CTA training with microinfusion of the appropriate ligand, and by microinfusing the ligand immediately before a CTA test and measuring subsequent immediate retrieval. No significant effect of the drug on the drinking and avoidance behavior was found in any of these tests under the conditions used in this study (Naor and Dudai 1996; Berman et al. 2000; Berman and Dudai 2001; data not shown).

\section{Data Analysis}

Differences among the groups were determined using ANOVA. For paired comparisons, Scheffe contrast tests were used with an $\alpha$ level of 0.05

\section{ACKNOWLEDGMENTS}

We thank Bob Rescorla for invaluable advice. This research was supported by grants from the Human Frontier Science Program, Volkswagen Stifftung, The Minerva Foundation, and the Israeli Science Foundation.

The publication costs of this article were defrayed in part by payment of page charges. This article must therefore be hereby marked "advertisement" in accordance with 18 USC section 1734 solely to indicate this fact.

\section{REFERENCES}

Aydin, A. and Pearce, J.M. 1997. Some determinants of response summation. Anim. Learn. Behav. 25: 108-121.

Berman, D.E. and Dudai, Y. 2001. Memory extinction, learning anew, and learning the new: Dissociations in the molecular machinery of learning in cortex. Science 291: 2417-2419.

Berman, D.E., Hazvi, S., Rosenblum, K., Seger, R., and Dudai, Y. 1998. Specific and differential activation of mitogen-activated protein kinase cascades by unfamiliar taste in the insular cortex of the behaving rat. J. Neurosci. 18: 10037-10044.

Berman, D.E., Hazvi, S., Neduva, V., and Dudai, Y. 2000. The role of identified neurotransmitter systems in the response of insular cortex to unfamiliar taste: Activation of ERK1-2 and formation of a memory trace. J. Neurosci. 20: 7017-7023.

Bonardi, C., Honey, R.C., and Hall, G. 1990. Context specificity of conditioning in flavor-aversion learning: Extinction and blocking tests. Anim. Learn. Behav. 18: 229-237.

Bouton, M.E. 1993. Context, time, and memory retrieval in the interference paradigms of Pavlovian learning. Psychol. Bull. 114: 80-99.

. 1994. Conditioning, remembering, and forgetting. J. Exp. Psychol. ABP 20: 219-231.

Bouton, M.E. and Swartzentruber, D. 1991. Sources of relapse after extinction in Pavlovian and instrumental learning. Clin. Psychol. Rev. 11: 123-140.

Brooks, D.C., Palmatier, M.I., Garcia, E., and Johnson, J.L. 1999. An extinction cue reduces spontaneous recovery of a conditioned taste aversion. Anim. Learn. Behav. 27: 77-88.

Bures, J., Bermudez-Rattoni, F., and Yamamoto, T. 1998. Conditioned taste aversion. Memory of a special kind. Oxford University Press, New York.

Christiansen, B.A. and Schmajuk, N.A. 1992. Hippocampectomy disrupts the topography of the rat eyeblink response during acquisition and extinction of classical conditioning. Brain. Res. 595: 206-214.

Ebbinghaus, H. [1885] 1964. Memory: A contribution to experimental psychology. Teachers College, Columbia University, NY, Dover, NY.

Falls, W.A., Miserendino, J.D., and Davis, M. 1992. Extinction of fear-potentiated startle: Blockade by infusion of an NMDA antagonist into the amygdala. J. Neurosci. 12: 854-863.

Ferry, B., Roozendaal, B., and McGaugh, J.L. 1999. Basolateral amygdala noradrenergic influences on memory storage as mediated by an interaction between $\beta$ - and $\alpha 1$-adrenoreceptors. J. Neurosci.. 19: 5119-5123.

Garcia, J., Ervin, F.R., and Koeling, R.A. 1966. Learning with prolonged delay of reinforcement. Psychon. Sci. 5: 121-122.

Garcia, K.S., Steele, P.M., and Mauk, M.D. 1999. Cerebellar cortex lesions prevent acquisition of conditioned eyelid responses. J. Neurosci. 19: 10940-10947.

Green, R.L. 1989. Spacing effects in memory: Evidence for a two-process account. J. Exp. Psych. Learn. Mem. Cogn. 15: 371-377.

Gutierrez, H., Hernandez-Echeagaray, E., Ramirez-Amaya, U., and Bermudez-Rattoni, F. 1999. Blockade of $N$-methyl-D-aspartate receptors in the insular cortex disrupts taste aversion and spatial memory formation. Neuroscience 89: 751-758.

Kamin, L.J. 1957. The retention of an incompletely learned avoidance response. J. Comp. Pbysiol. Psychol. 50: 457-460.

Kraemer, P.J. and Spear, N.E. 1992. The effect of nonreinforced stimulus exposure on the strength of a conditioned taste aversion as a function of retention interval: Do latent inhibition and extinction involve a shared process? Anim. Learn. Behav. 20: 1-7.

LaBar, K.S., Gatenby, J.C., Gore, J.C., LeDoux, J.E., and Phelps, E.A. 1998. Human amygdala activation during conditioned fear acquisition and extinction: A mixed-trial fMRI study. Neuron 20: 937-945.

Lamprecht, R., Hazvi, S., and Dudai, Y. 1997. cAMP response element-binding protein in the amygdala is required for long- but not short-term conditioned taste aversion memory. J. Neurosci. 17: 8443-8450.

Lee, H. and Kim, J.J. 1998. Amygdalar NMDA receptors are critical for new fear learning in previously fear-conditioned rats. J. Neurosci. 18: 8444-8454.

Mackintosh, N.J. 1983. Conditioning and associative learning. Oxford University Press, Oxford, UK.

McGaugh, J.L. 1966. Time-dependent processes in memory storage. Science 153: 1351-1358.

Naor, C. and Dudai, Y. 1996. Transient impairment of cholinergic function in the rat insular cortex disrupts the encoding of taste in conditioned taste aversion. Behav. Brain. Res. 79: 61-67.

Pavlov, I.P. 1927. Conditioned reflexes. In An investigation of the physiological activity of the cerebral cortex, Oxford University Press, London, UK.

Paxinos, G. and Watson, C. 1998. The rat brain in stereotaxic coordinates. Fourth ed. Academic Press, San Diego, CA.

Pereyra, P., Portino, R.E.G., and Maldonado, H. 2000. Long-lasting and context-specific freezing preference is acquired after spaced repeated presentations of a danger stimulus in the crab Chasmagnathus. Neurobiol. Learn. Mem. 74: 119-134.

Przybyslawski, J., Roullet, P., and Sara, S. 1999. Attenuation of emotional and nonemotional memories after their reactivation: Role of $\beta$-adrenergic receptors. J. Neurosci. 19: 6623-6628.

Quirk, G.J., Russo, G.K., Barron, J.L., and Lebron, K. 2000. The role of

\section{$\begin{array}{llllllllllllllllllllllllll}L & E & A & R & N & I & N & G & \mathbf{Z} & M & E & M & O & R & Y\end{array}$


ventromedial prefrontal cortex in the recovery of extinguished fear. $J$. Neurosci. 20: 6225-6231.

Rescorla, R.A. 1979. Conditioned inhibition and extinction. In: Mechanisms of learning and motivation: A memorial volume to Jerzy Konorski (eds. A. Dickinson and R.A. Boakes), pp. 83-110. LEA, Hillsdale, NJ.

1996. Preservation of Pavlovian associations through extinction. $Q$. J. Exp. Psych. 49B: 245-258.

Rescorla, R.A. and Heth, C.D. 1975. Reinstatement of fear to an extinguished conditioned stimulus. J. Exp. Psychol. ABP 1: 88-96.

Rosas, J.M. and Bouton, M.E. 1996. Spontaneous recovery after extinction of a conditioned taste aversion. Anim. Learn. Behav. 24: 341-348.

Rosenblum, K., Meiri, N., and Dudai, Y. 1993. Taste memory: The role of protein synthesis in gustatory cortex. Behav. Neural. Biol. 59: 49-56.

Rosenblum, K., Berman, D.E., Hazvi, S., Lamprecht, R., and Dudai, Y. 1997. NMDA receptor and the tyrosine phosphorylation of its $2 \mathrm{~B}$ subunit in taste learning in the rat insular cortex. J. Neurosci. 17: 5129-5135.

Swank, M.W., Ellis, A.E., and Cochran, B.N. 1996. c-Fos antisense blocks acquisition and extinction of conditioned taste aversion in mice. Neuroreport 7: 1866-1870.

Weible, A.P., McEchron, M.D., and Disterhoft, J.F. 2000. Cortical involvement in acquisition and extinction of trace eyeblink conditioning. Behav. Neurosci. 114: 1058-1067.

Wik, G., Elbert, T., Fredrikson, M., Hoke, M., and Ross, B. 1997. Magnetic brain imaging of extinction processes in human classical conditioning Neuroreport 8: 1789-1792.

Yasoshima, Y., Morimoto, T., and Yamamoto, T. 2000. Different disruptive effects on the acquisition and expression of conditioned taste aversion by blockades of amygdalar ionotropic and metabotropic glutamatergic receptor subtypes in rats. Brain Res. 869: 15-24.

Yin, J.C.P., Del Vecchio, M., Zhou, H., and Tully, T. 1995. CREB as a memory modulator: Induced expression of a $A C R E B 2$ activator isoform enhances long-term memory in Drosophila. Cell 81: 107-115.

Received July 16, 2002; accepted in revised form November 15, 2002.

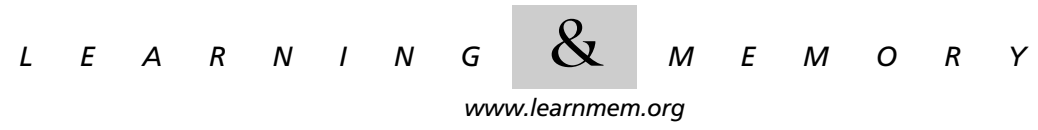




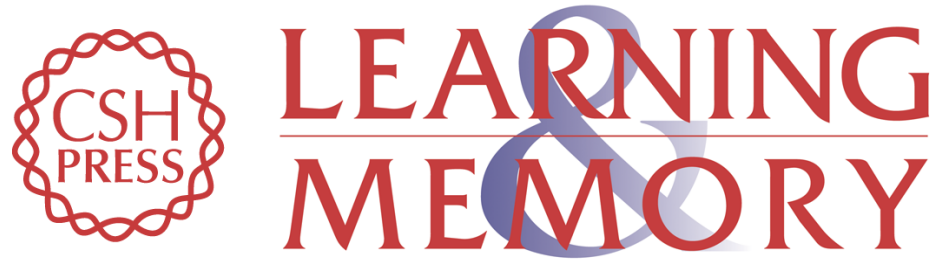

\section{Conflicting Processes in the Extinction of Conditioned Taste Aversion: Behavioral and Molecular Aspects of Latency, Apparent Stagnation, and Spontaneous Recovery}

Diego E. Berman, Shoshi Hazvi, Jimmy Stehberg, et al.

Learn. Mem. 2003, 10:

Access the most recent version at doi:10.1101//m.53703

References This article cites 32 articles, 12 of which can be accessed free at:

http://learnmem.cshlp.org/content/10/1/16.full.html\#ref-list-1

License

Email Alerting Receive free email alerts when new articles cite this article - sign up in the box at the Service top right corner of the article or click here. 